\title{
IDIOPATHIC RECURRENT PERICARDITIS REFRACTORY TO COLCHICINE TREATMENT CAN REVEAL TUMOR NECROSIS FACTOR RECEPTOR-ASSOCIATED PERIODIC SYNDROME
}

\author{
L. CANTARINI, O.M. LUCHERINI ${ }^{1}$, R. CIMAZ ${ }^{2}$, C.T. BALDARI ${ }^{1}$, F. BELLISAI, \\ S. ROSSI PACCANI' , F. LAGHI PASINI ${ }^{3}$, P.L. CAPECCHI ${ }^{3}$, G.D. SEBASTIANI ${ }^{4}$ and M. GALEAZZI
}

Interdepartmental Research Center of Systemic Autoimmune and Autoinflammatory Diseases, Unit of Rheumatology, Policlinico Le Scotte, University of Siena, Siena; 'Department of Evolutionary Biology, University of Siena, Siena; ' Department of Paediatrics, Rheumatology Unit, Anna Meyer Children's Hospital and University of Florence; ${ }^{3}$ Interdepartmental Research Center of Systemic Autoimmune and Autoinflammatory Diseases, Unit of Immunological Sciences, Policlinico Le Scotte, University of Siena, Siena; ${ }^{4}$ UOC of Rheumatology, S. Camillo-Forlanini Hospital, Rome, Italy

$$
\text { Received May 8, } 2009 \text { - Accepted August 25, } 2009
$$

The first two authors contributed equally to this study

Recurrences develop in up to $\mathbf{2 0 - 5 0 \%}$ of patients with acute pericarditis. Although different causes of recurrent pericarditis have been identified, the etiology remains obscure in most cases which are therefore labelled as idiopathic. Autoinflammatory syndromes include familial Mediterranean fever (FMF), due to mutations in the $M E F V$ gene, and tumor necrosis factor receptor-associated periodic syndrome (TRAPS), due to mutations in the TNFRSF1A gene. Recurrent pericarditis is a common feature of both conditions, but it rarely occurs alone. Colchicine is the standard treatment for FMF, while patients with TRAPS do not respond to colchicine therapy, but are responsive to corticosteroids. Based on the proven efficacy of colchicine in preventing polyserositis in FMF, colchicine has been proposed for the treatment of recurrent pericarditis and is able to decrease the recurrence rate. Our aim was to investigate the possible involvement of TNFRSF1A mutations in a group of patients with idiopathic recurrent pericarditis who were refractory to colchicine treatment. Thirty consecutive patients (17 males, 13 females) diagnosed with idiopathic recurrent pericarditis, who were characterized by a poor response to colchicine treatment, were enrolled in the study. Mutations of the TNFRSF1A gene were searched for by amplifying, using polymerase chain reaction (PCR), genomic DNA, and direct sequencing. TNFRSF1A mutations were found in 4 of the 30 patients. None of these 4 patients had a family history of recurrent inflammatory syndromes or history of pericarditis. One of the 4 patients had a novel heterozygous deletion $(\Delta$ Y103-R104) and three patients carried a heterozygous low-penetrance R92Q mutation. Our data suggest that TRAPS should be kept in mind in the differential diagnosis of recurrent pericarditis, and mutation analysis of the TNFRSF1A gene should be considered, in addition to $M E F V$ analysis, in patients of Mediterranean origin. A poor response to colchicine treatment and/or a steroid-dependence may be the clue to investigate TNFRSF1A mutations in patients with idiopathic recurrent pericarditis.

Key words: idiopathic recurrent pericarditis, TNFRSF1A mutation,

$$
\text { tumor necrosis factor receptor-1 associated periodic syndrome, colchicine resistance, etanercept }
$$

Mailing address: Dr Luca Cantarini,

Institute of Rheumatology,

Policlinico "Le Scotte",

0394-6320 (2009)

University of Siena,

Viale Bracci 1, 53100 Siena, Italy

Tel: ++393479385457 Fax: ++3957740450

e-mail: cantariniluca@hotmail.com

Copyright $\odot$ by BIOLIFE, s.a.s.

This publication and/or article is for individual use only and may not be further

1051 reproduced without written permission from the copyright holder. 
Recurrences develop in $20-50 \%$ of patients with acute pericarditis (1). Clinical signs and symptoms of acute pericarditis are characteristic of pericardial disease and include elevation of inflammatory markers, precordial pain, fever, pericardial friction rub, dyspnoea, and electrocardiogram changes. Clinical manifestations during recurrences are similar to the first episode, but the subsequent episodes are typically milder and may be limited to precordial pain only. The number of recurrences and the intervals between attacks can vary among patients. Recurrent pericarditis may be isolated or associated with autoimmune rheumatic diseases, viral or bacterial infections, and neoplastic diseases (2). Although many causes have been identified, the etiology of recurrent pericarditis remains obscure in most cases (2).

Recurrent pericarditis, in the form of recurrent polyserositis, is common in familial Mediterranean fever (FMF) (3) and other auto-inflammatory disorders, a group of diseases caused by primary dysfunction of the innate immune system and characterized by seemingly unprovoked attacks of fever in association with localised inflammation that can affect multiple organ systems (4).

Tumor necrosis factor receptor-1-associated periodic syndrome (TRAPS) is the most common auto-inflammatory autosomal dominant disorder. TRAPS is caused by mutations in the gene TNFRSF1A, located on chromosome 12p13, encoding the $55-\mathrm{kD}$ receptor for tumor necrosis factor- $\alpha$ (TNF- $\alpha)(5)$. The disease is characterized by recurrent attacks of fever typically lasting from 1 to 3 weeks; in addition to fever, common clinical features include mainly periorbital oedema, a migratory erythematous plaque simulating erysipela with underlying myalgia, and arthritis or arthralgia; serous membrane inflammation is also possible (67). Patients with TRAPS, unlike those with FMF, respond poorly to colchicine treatment, but are responsive to corticosteroids (8).

Although few patients with isolated serosal pericardial involvement carrying mutations in TNFRSF1A have been described (6), to the best of our knowledge no studies have been published on TNFRSF1A gene mutations in patients with idiopathic recurrent pericarditis. Our aim is to investigate the possible involvement of TNFRSF1A gene mutations in a group of patients with idiopathic recurrent pericarditis, who were refractory to colchicine treatment.

\section{MATERIALS AND METHODS}

\section{Patients}

We enrolled 30 consecutive patients (17 males, 13 females) diagnosed with idiopathic recurrent pericarditis who were characterized by a poor response to colchicine treatment at standard dose (1-2 mg/day). All patients were seen between September 2005 and November 2008 in our Institution, and were part of a larger cohort of patients treated for idiopathic recurrent pericarditis. Table I summarizes the main characteristics of the cohort of patients enrolled in our study.

Each patient underwent detailed examinations in order to rule out underlying diseases such as infections, connective tissue disorders and malignancies. None of the patients' past medical history was relevant for cardiac surgery or radiation therapy. Criteria for the diagnosis of recurrent pericarditis were: a) a documented first attack of acute pericarditis according to accepted diagnostic criteria (fever, pericardial friction rub, electrocardiographic changes, echocardiographic evidence of pericardial effusion, increases in the white blood cell count and/or erythrocyte sedimentation rate (ESR) and/or C-reactive protein (CRP) and/or serum amyloid A (SAA); and b) evidence of at least one recurrence.

Recurrence was documented by pain and one or more of the following signs: fever, pericardial friction rub, electrocardiographic changes, echocardiographic evidence of pericardial effusion, and elevations in the white blood cell count or ESR or CRP or SAA. Resistance to colchicine was defined as an unfavorable clinical response with persistence or recurrence of fever and pericardial effusion appearance or worsening during treatment. All patients were Caucasians of Italian ancestry; they all provided written consent for genetic testing, in accordance with the Helsinki Declaration and local Ethical Committee regulations.

\section{DNA extraction}

Mononuclear cells were purified from peripheral blood from healthy donors and patients (with informed consent) by density gradient centrifugation on FicollPaque (Amersham Biosciences, Buckinghamshire, UK), using a Beckman GS-6R tabletop centrifuge (Beckman Coulter SpA, Milan, Italy). Cells were washed $2 \mathrm{X}$ in phosphate buffered saline (PBS), resuspended in RPMI 1640 (Invitrogen Ltd, Paisley, UK) (buffered with sodium bicarbonate to $\mathrm{pH} 7.2$ ) supplemented with $7.5 \%$ fetal calf serum (FCS) (Hyclone, Thermofischer Scientific Inc, SouthLogan, UT), plated in plastic flasks (Sarstedt AG, 
A)

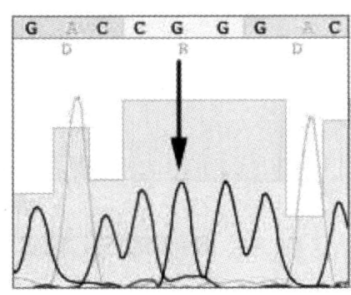

WT/WT

B)

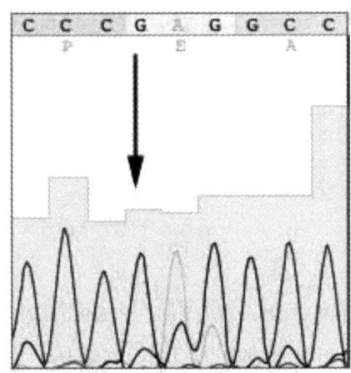

WT/WT
Patient
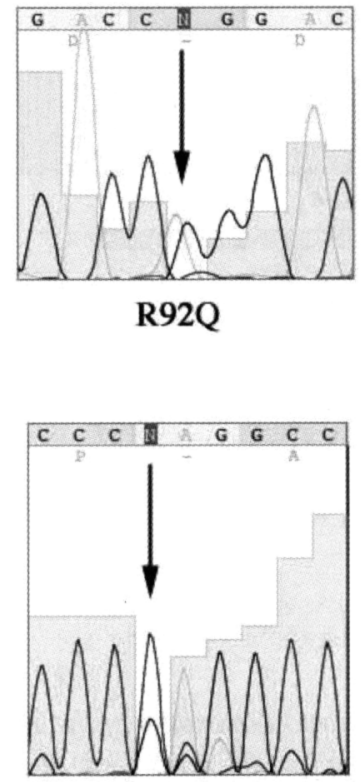

E148Q

Fig. 1. A) DNA sequence electropherograms demonstrating the R92Q mutation in TNFRSF1A. WT, wild type. Sequence electropherograms of TNFRSFIA exon 4 PCR amplification products from healthy donor and patient. The arrow indicates the heterozygous $G / A$ substitution resulting in the exchange of arginine for glutamine at position 92 . B) DNA sequence electropherograms demonstrating the E148Q mutation in MEFV.WT, wild type. Sequence electropherograms of MEFV exon 2 PCR amplification products from healthy donor and patient. The arrow indicates the heterozygous $G / C$ substitution resulting in the exchange of glutamic acid for glutamine at position 148.

Numbrecht, Germany) and incubated overnight at $37^{\circ} \mathrm{C}$ in a humidified atmosphere with $5 \% \mathrm{CO}_{2}$. Non-adherent cells, which consisted principally of peripheral blood lymphocytes $(\mathrm{PBL})$, were centrifuged and resuspended in fresh RPMI 1640 supplemented with $7.5 \%$ FCS. Genomic DNA was isolated from peripheral blood lymphocytes of patients and healthy controls using QIAamp DNA mini Kit (Qiagen, Hilden Germany).

\section{Genomic DNA amplification and mutation detection}

The TNFRSFIA gene exons 2, 3, 4 and 6, which encode for the extracellular domain of the $55-\mathrm{kD}$ receptor for TNF- $\alpha$ and the $M E F V$ gene exons 2, 3, and 10, where the majority of known mutations are found, were amplified by polymerase chain reaction (PCR) using Expand High Fidelity PCR System (Roche, Germany). PCR products were purified using Wizard SV Gel and PCR Clean-Up System (Promega, Madison, WI, USA). Sequencing was carried out on the ABI 3730 DNA analyzer (Bio-Fab Research srl, Italy) using the same primers as those used in the PCR.

\section{Restriction analysis of the Y103-R104 deletion}

PCR of TNFRSFIA (exon 4) was performed in healthy donor and patient. The 332 basepair amplicons were then digested by RsaI restriction enzyme (Fermentas) according to the manufacturer's instructions.

\section{RESULTS}

Out of 30 patients tested, four carried a mutation in the TNFRSF1A gene. Three of these (patients 12, 17 and 26 in Table I) carried a heterozygous lowpenetrance R92Q mutation (Fig. 1A), and one (patient 4 in Table I) carried a novel heterozygous deletion ( $\Delta$ Y103-R104) (Fig. 2). The presence of the Y103R104 deletion was further confirmed by restriction analysis carried out with RsaI restriction enzyme; in fact, deletion destroys an RsaI restriction site (GT $\downarrow$ 

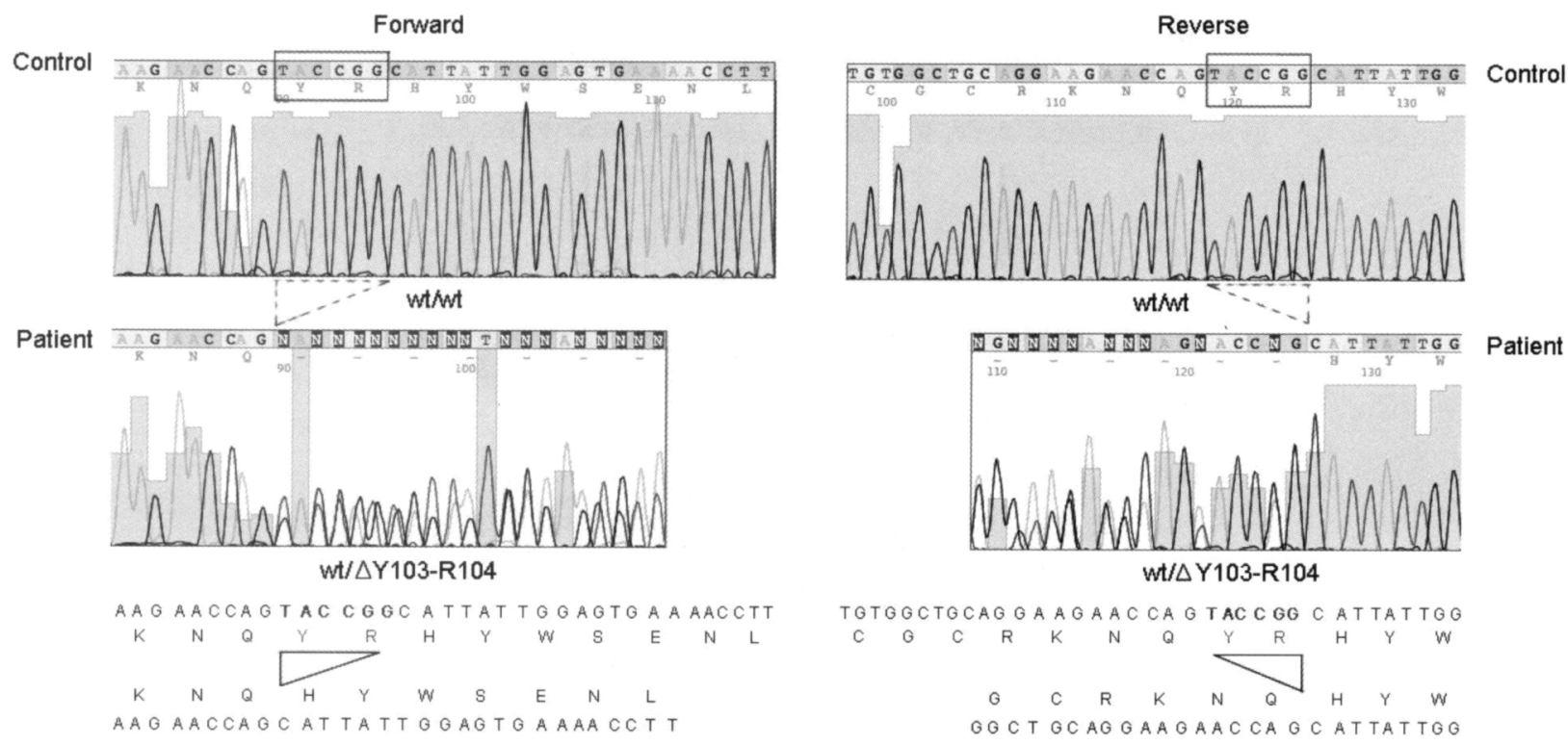

Fig. 2. Identification of novel mutation in exon 4 of the TNFRSF1A gene. DNA Sequence electropherograms of TNFRSF1A exon 4 PCR amplification product in forward (left) and reverse (right) from healthy donor (top) and patient (bottom). The patient presents six nucleotide heterozygous deletion (TACCGG nucleotide sequence) resulting in an electroferogram shift. This deletion causes the loss of tyrosine and arginine aminoacid at position 103 and 104, respectively ( $\Delta Y 103-$ R104).

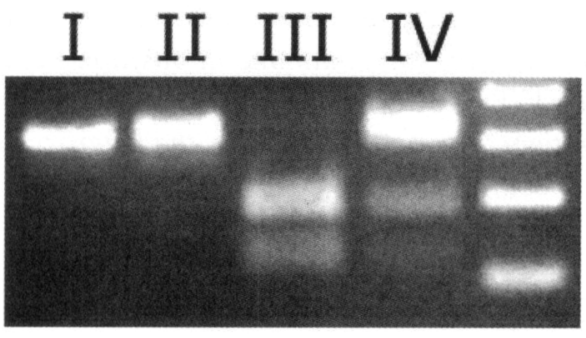

Fig. 3. Restriction analysis of the Y103-R104 deletion by RsaI restriction enzyme. PCR amplification TNFRSFIA exon 4 was performed in healthy donor (I) and patient (II). PCR product digestion by Rsal restriction enzyme resulted in two bands of 145 and 187 base pairs and three bands of 145, 187 and 332 base pairs in healthy donor (III) and patient (IV), respectively.

AC) (Fig. 3). All four patients were diagnosed with TRAPS. The mother, father and brother of patient 4 were clinically healthy and did not carry the mutation, indicating that the mutation is likely to have occurred de novo. The father of patient 12 , the father of patient 17 and the mother of patient 26 also carried the mutation R92Q, but they were all clinically healthy. Patient 12 also carried a low-penetrance mutation E148Q in the
$M E F V$ gene (Fig. 1B), not carried by the parents, but was clinically diagnosed with TRAPS based on poor response to colchicine, the duration of fever attacks ( $>$ 2 weeks), and late onset of symptoms (34 years).

These four patients, who, like all the others in the study, had a poor response to colchicine, were selected for treatment with the tumor necrosis factor (TNF)-a neutralizing agent, etanercept ( $25 \mathrm{mg}$ twice weekly). Three additional patients who did not carry mutations had increased ( $>100 \mathrm{mg} / \mathrm{L}$ ) levels of circulating SAA, measured at least three times also during symptom free intervals. Two of them (patients 9 and 13 in Table I) were successfully treated with anakinra (100 mg/daily), a recombinant human interleukin-1 receptor antagonist, while the third patient's parents refused this off-label treatment. In the remaining cases indomethacin $(50-150 \mathrm{mg} /$ daily) was added to colchicine. In a few patients (patients $11,16,19$ and 21 in Table I) a further addition of low-dose prednisone was required in order to prevent recurrences. Eventually, all patients achieved good disease control. At the time of genetic testing all patients were taking colchicine 1-2 mg/daily. 
Table I. Demographic, genetic and laboratory characteristics of patients.

\begin{tabular}{|c|c|c|c|c|c|}
\hline $\mathbf{P t}$ & $\begin{array}{c}\text { Age at testing/Age at onset } \\
\text { (yrs) }\end{array}$ & Sex & $\begin{array}{c}\mathbf{S A A} \\
(\mathrm{mg} / \mathrm{L})\end{array}$ & $\begin{array}{c}\text { Mutations in } \\
\text { TNFRSF1A }\end{array}$ & $\begin{array}{c}\text { Mutations in } \\
M E F V\end{array}$ \\
\hline 1 & $14 / 12$ & $\mathrm{~F}$ & 158 & - & - \\
\hline 2 & $28 / 27$ & $\mathrm{~F}$ & 58 & - & - \\
\hline 3 & $37 / 35$ & $\mathrm{M}$ & 28 & - & - \\
\hline 4 & $28 / 13$ & $\mathrm{M}$ & 181 & Delta Y103-R104 heterozygous & - \\
\hline 5 & $26 / 22$ & $\mathrm{M}$ & 42 & - & - \\
\hline 6 & $42 / 35$ & $\mathrm{~F}$ & 6 & - & - \\
\hline 7 & $22 / 17$ & $\mathrm{M}$ & 8 & - & - \\
\hline 8 & $34 / 31$ & $\mathrm{M}$ & 42 & - & - \\
\hline 9 & $27 / 24$ & $\mathrm{~F}$ & 146 & - & - \\
\hline 10 & $39 / 36$ & $\mathrm{~F}$ & 44 & - & - \\
\hline 11 & $59 / 51$ & $\mathrm{~F}$ & 88 & - & - \\
\hline 12 & $50 / 34$ & $\mathrm{M}$ & 138 & R92Q heterozygous & E148Q homozygous \\
\hline 13 & $56 / 49$ & $\mathrm{M}$ & 128 & - & - \\
\hline 14 & $32 / 29$ & $\mathrm{~F}$ & 16 & - & - \\
\hline 15 & $29 / 24$ & $\mathrm{M}$ & 34 & - & - \\
\hline 16 & $48 / 43$ & $\bar{M}$ & 68 & - & - \\
\hline 17 & $32 / 28$ & $\mathrm{~F}$ & 112 & R92Q heterozygous & - \\
\hline 18 & $16 / 13$ & $\mathrm{M}$ & 26 & - & - \\
\hline 19 & $36 / 35$ & $\mathrm{~F}$ & 98 & - & - \\
\hline 20 & $24 / 21$ & $\mathrm{M}$ & 48 & - & - \\
\hline 21 & $36 / 32$ & $\mathrm{M}$ & 67 & - & - \\
\hline 22 & $41 / 37$ & $\mathrm{~F}$ & 59 & - & - . \\
\hline 23 & $50 / 54$ & $\mathrm{M}$ & 76 & - & - \\
\hline 24 & $23 / 22$ & $\mathrm{M}$ & 8 & - & - \\
\hline 25 & $15 / 13$ & $\mathrm{M}$ & 2 & - & - \\
\hline 26 & $41 / 38$ & $F$ & 121 & R92Q heterozygous & - \\
\hline 27 & $53 / 50$ & $\mathrm{~F}$ & 9 & - & - \\
\hline 28 & $29 / 28$ & $\mathrm{M}$ & 39 & - & - \\
\hline 29 & $34 / 32$ & $\mathbf{M}$ & 24 & - & - \\
\hline 30 & $17 / 16$ & $\mathrm{~F}$ & 26 & - & - \\
\hline
\end{tabular}

The table summarizes data from the entire cohort of patients enrolled. Gender, age at disease onset, age at the time of genetic testing and the circulating levels of serum amyloid $A$ are shown. The results of genetic testing for mutations of the gene responsible for the tumor necrosis factor- $\alpha$ receptor-associated periodic syndrome (TNFRSF1A gene) and of the familial Mediterranean fever (MEFV gene) are also listed. List of abbreviations: Pt $=$ patient; yrs $=y e a r s ; ~ S A A=$ Serum Amyloid $A ; F=$ female; $M=$ male

After one year of follow-up the four patients with TRAPS who were treated with etanercept did not show signs of disease relapse, and in all four, SAA levels had already decreased to normal values a few weeks after the initiation of treatment. The same was true for the 2 patients treated with anakinra. 


\section{DISCUSSION}

Recurrences develop in $20-50 \%$ of adult patients with acute pericarditis (1). The aetiology of acute pericarditis and the cause of the recurrences remain obscure in most cases, which are thus labeled as idiopathic. Autoimmune responses may play a role in the pathogenesis of recurrent pericarditis (9). Other mechanisms that have been hypothesized in the pathogenesis include insufficient treatment during the first episode, possible re-infections and augmented viral DNA/RNA replication in pericardial tissue as possible consequences of longterm treatment with steroids (10).

Recurrent pericarditis and other recurrent serositis, often in the form of polyserositis, are common in FMF and TRAPS $(3,6-7)$; furthermore, patients with isolated recurrent pericarditis have been reported both in FMF (11) and in TRAPS (6). Dodè et al. recently described two patients presenting recurrent pericarditis as the sole clinical manifestation of TRAPS and carrying the low-penetrance mutations R92Q and P46L. In FMF, the incidence of pericardial involvement has been reported in $1.4 \%$ of cases in a series (12), but the incidence rises to $27 \%$ in echocardiographic studies (3).

Recently, Brucato et al. investigated the presence of FMF in two series of Italian patients with recurrent pericarditis; mutations in $M E F V$ were searched for respectively in 23 and 61 patients, but never found $(1,13)$. In neither trial was genetic testing for mutations in the TNFRSF1A gene carried out. In our study we found a high incidence of TNFRSF1A mutations, in comparison to the absence of $M E F V$ mutations, which may be due to the inclusion criteria and demographic characteristics of patients: 1) the enrolment of patients unresponsive to colchicine, which ruled out the majority of FMF patients that are typically responsive to colchicine $(14) ; 2)$ the age of disease onset in most cases in our series was $>20$ years, a highly unlikely occurrence in FMF.

TNFRSF1A is a transmembrane glycoprotein consisting of an extracellular region characterized by a distinct pattern of 4 cysteine-rich domains (CRDs 1-4), a transmembrane region, and an intracellular death domain (DD), which promotes TNFRSF1A signaling (15) The binding site for TNF $\alpha$ is formed by CRD2 and CRD3 (16) while CRD1 is the pre- ligand assembly binding domain and is thought to mediate TNFRSF1A self-assembly (17). More than 40 individual mutations have been identified, the majority of which is localised in the CRD1 and CRD2 domains. Most mutations described involve cysteine residues and are associated with a higher disease penetrance; however several variants involving other residues have been reported (18). In vitro studies suggest that these TRAPS-associated TNFRSF 1A mutations are responsible for altered TNFRSF1A expression, impaired TNF- $\alpha$ binding and shedding (5), defective trafficking (19-20) and abnormal apoptosis (21-22).

Three of our patients carried the R92Q mutation in the TNFRSF $1 A$ gene and patient 4 carried a novel deletion ( $\triangle Y 103-R 104)$ in the CRD3 domain of TNFRSF1A that might be involved in receptor shedding, thus affecting TNFRSF1A membrane localization, or other receptor functions. The actual functional implication of this deletion is under investigation. The $\mathrm{R} 92 \mathrm{Q}$ mutation is a low-penetrance allele common in patients of Mediterranean origin and has been found in $1.8 \%$ of Caucasian patients with recurrent inflammatory syndromes. R92Q mutation may cause a milder disease and may often be associated with an oligosymptomatic course $(7,23)$. The clinical phenotype of TRAPS is expanding, and since the discovery of TNFRSF1A, several studies have indicated that the low-penetrance TNFRSF1A variants might contribute to atypical inflammatory responses in TRAPS, including cardiac diseases (myocarditis and pericarditis) $(6,24)$, as in three of our cases.

Based on the proven efficacy of colchicine in preventing polyserositis in FMF, colchicine has been proposed for the treatment of recurrent pericarditis and is able to decrease the recurrence rate (25). However, patients with TRAPS are characterized by a poor response to colchicine and are typically responsive to corticosteroid administration (8), although steroids do not seem to protect completely from the risk of amyloidosis which occurs in up to $25 \%$ of patients (26). Although TNF- $\alpha$ levels are not usually significantly elevated in TRAPS patients compared with healthy controls, etanercept has been shown to elicit a beneficial response in patients with TRAPS-related amyloidosis (27); it can reduce 
corticosteroid requirement and also prevent flare-ups (28). Accordingly, our patients were successfully treated with etanercept.

The patients who did not carry mutations, but persistently showed elevated circulating SAA levels, were considered to be affected by an unrecognized autoinflammatory disorder, since a large percentage of these patients demonstrated an elevated acute phase response between clinically symptomatic attacks (7). The choice of anakinra in our 2 patients proved successful, in accordance with recent published data (29), and was based on the demonstrated efficacy of anakinra in the treatment of a large spectrum of autoinflammatory diseases (30-31).

In conclusion, our preliminary data suggest that TRAPS should be kept in mind in the differential diagnosis of recurrent pericarditis, and mutation analysis in TNFRSF1A should be considered, in addition to $M E F V$ analysis, in patients of Mediterranean origin. A poor response to colchicine treatment and/or a steroid-dependence may be the clue to investigate $T N F R S F 1 A$ mutations in patients with idiopathic recurrent pericarditis. Furthermore, the discovery of TNFRSF1A mutations may have relevant clinical implications for patients, since etanercept is still to be considered the first-line therapy for TRAPS, although its long-term benefit has yet to be fully elucidated.

\section{REFERENCES}

1. Brucato A, Brambilla G, Moreo A, et al. Long-term outcomes in difficult-to-treat patients with recurrent pericarditis. Am J Cardiol 2006; 98:267-71.

2. Imazio M, Cecchi E, Demichelis B, et al. Indicators of poor prognosis of acute pericarditis. Circulation 2007; 115:2739-44.

3. Dabestani A, Noble LM, Child JS, Krivokapich $\mathrm{J}$, Schwabe AD. Pericardial disease in familial Mediterranean fever: an echocardiographic study. Chest 1982; 81:592-5.

4. Ozen S, Hoffman HM, Frenkel J, Kastner D. Familial Mediterranean fever (FMF) and beyond: a new horizon. Fourth International Congress on the Systemic Autoinflammatory Diseases held in Bethesda, USA, 6-10 November 2005. Ann Rheum Dis 2006; 65:961-4.

5. McDermott MF, Aksentijevich I, Galon J, McDermott
EM, Ogunkolade BW, Centola M, et al. Germline mutations in the extracellular domains of the $55 \mathrm{kDa}$ TNF receptor, TNFR 1 , define a family of dominantly inherited autoinflammatory syndromes. Cell 1999; 97:133-44.

6. Dodé $\mathrm{C}$, André $\mathrm{M}$, Bienvenu $\mathrm{T}$, et al; French Hereditary Recurrent Inflammatory Disorder Study Group. The enlarging clinical, genetic, and population spectrum of tumor necrosis factor receptor-associated periodic syndrome. Arthritis Rheum 2002; 46:2181-8.

7. Hull KM, Drewe E, Aksentijevich I, et al. The TNF receptor-associated periodic syndrome (TRAPS): emerging concepts of an autoinftammatory disorder. Medicine (Baltimore) 2002; 81:349-68.

8. McDermott EM, Smillie DM, Powell RJ. Clinical spectrum of familial Hibernian fever: a 14-year follow-up study of the index case and extended family. Mayo Clin Proc 1997; 72:806-17.

9. Spodick DH. The pericardium: a comprehensive textbook. New York: Marcel Dekker; 1997. p. 26090.

10. Lange RA, Hillis LD. Acute pericarditis. N Engl J Med 2004; 351:2195-202.

11. Tutar EH, Imamoglu A, Atalay S. Recurrent pericarditis as a manifestation of familial Mediterranean fever. Circulation 2000; 101:E71-2.

12. Tunca M, Akar S, Onen F, et al; Turkish FMF Study Group. Familial Mediterranean fever (FMF) in Turkey: results of a nationwide multicenter study. Medicine (Baltimore) 2005; 84:1-11.

13. Brucato $A$, Shinar $Y$, Brambilla $G$, et al. Idiopathic recurrent acute pericarditis: familial Mediterranean fever mutations and disease evolution in a large cohort of Caucasian patients. Lupus 2005; 14:670-4.

14. Zemer D, Pras M, Sohar E, Modan M, Cabili S, Gafni J. Colchicine in the prevention and treatment of the amyloidosis of familial Mediterranean fever. N Engl J Med 1986; 314:1001-5.

15. Hehlgans $T$, Pfeffer $K$. The intriguing biology of the tumour necrosis factor/tumour necrosis factor receptor superfamily: players, rules and the games. Immunology 2005; 115:1-20.

16. Banner DW, D'Arcy A, Janes W, et al. Crystal structure of the soluble human $55 \mathrm{kd}$ TNF receptorhuman TNF beta complex: implications for TNF 
receptor activation. Cell 1993; 73:431-45.

17. Chan FK, Chun HJ, Zheng L, Siegel RM, Bui $\mathrm{KL}$, Lenardo MJ. A domain in TNF receptors that mediates ligand-independent receptor assembly and signaling. Science 2000; 288:2351-4.

18. Aksentijevich I, Galon J, Soares M, et al. The tumor-necrosis-factor receptor-associated periodic syndrome: new mutations in TNFRSF1A, ancestral origins, genotype-phenotype studies, and evidence for further genetic heterogeneity of periodic fevers. Am J Hum Genet 2001; 69:301-14.

19. Lobito AA, Kimberley FC, Muppidi JR, et al. Abnormal disulfide-linked oligomerization results in ER retention and altered signaling by TNFR1 mutants in TNFR1-associated periodic fever syndrome (TRAPS). Blood 2006; 108:1320-7.

20. Nedjai B, Hitman GA, Yousaf N, et al. Abnormal tumor necrosis factor receptor I cell surface expression and NF-kappaB activation in tumor necrosis factor receptor-associated periodic syndrome. Arthritis Rheum 2008; 58:273-83.

21. D'Osualdo A, Ferlito F, Prigione I, et al. Neutrophils from patients with TNFRSF1A mutations display resistance to tumor necrosis factor-induced apoptosis

- Pathogenetic and clinical implications. Arthritis Rheum 2006; 54:998-1008.

22. Nedjai B, Hitman GA, Quillinan $\mathrm{N}$, et al. Proinflammatory action of the anti-inflammatory drug infliximab in tumor necrosis factor receptorassociated periodic syndrome. Arthritis Rheum 2009; 60:619-25.

23. Ravet $\mathrm{N}$, Rouaghe $\mathrm{S}$, Dodé $\mathrm{C}$, et al. Clinical significance of $\mathrm{P} 46 \mathrm{~L}$ and $\mathrm{R} 92 \mathrm{Q}$ substitutions in the tumour necrosis factor superfamily 1 A gene. Ann Rheum Dis 2006; 65:1158-62.

24. Trost S, Rosé CD. Myocarditis and sacroiliitis: 2 previously unrecognized manifestations of tumor necrosis factor receptor associated periodic syndrome. J Rheumatol 2005; 32:175-7.
25. Imazio $\mathrm{M}$, Bobbio $\mathrm{M}$, Cecchi $\mathrm{E}$, et al. Colchicine as first-choice therapy for recurrent pericarditis: results of the CORE (COlchicine for Recurrent pericarditis) trial. Arch Intern Med 2005; 165:1987-91.

26. Aganna E, Hawkins PN, Ozen S, et al. Allelic variants in genes associated with hereditary periodic fever syndromes as susceptibility factors for reactive systemic AA amyloidosis. Genes Immun 2004; 5: 289-93.

27. Kallinich T, Briese S, Roesler J, et al. Two familial cases with tumor necrosis factor receptor-associated periodic syndrome caused by a non-cysteine mutation (T50M) in the TNFRSF1A gene associated with severe multiorganic amyloidosis. J Rheumatol 2004; 31:2519-22.

28. Drewe E, McDermott EM, Powell PT, Isaacs JD, Powell RJ. Prospective study of anti-tumour necrosis factor receptor superfamily $1 \mathrm{~B}$ fusion protein, and case study of anti-tumour necrosis factor receptor superfamily $1 \mathrm{~A}$ fusion protein, in tumour necrosis factor receptor associated periodic syndrome (TRAPS): clinical and laboratory findings in a series of seven patients. Rheumatology (Oxford) 2003; 42: 235-9.

29. Picco P, Brisca G, Traverso F, Loy A, Gattorno M, Martini A. Successful treatment of idiopathic recurrent pericarditis in children with interleukinlbeta receptor antagonist (anakinra): An unrecognized autoinflammatory disease? Arthritis Rheum 2009; 60:264-8.

30. Kuijk LM, Govers AM, Frenkel J, Hofhuis WJ. Effective treatment of a colchicine-resistant familial Mediterranean fever patient with anakinra. Ann Rheum Dis 2007; 66:1545-6.

31. Goldbach-Mansky R, Dailey NJ, Canna SW, Gelabert A, Jones J, Runin BI, et al. Neonatal-onset multisystem inflammatory diseases responsive to interleukin-1 inhibition. N Engl J Med 2006; 355: 581-92. 